International Journal of Medical Anesthesiology 2021; 4(3): 41-46

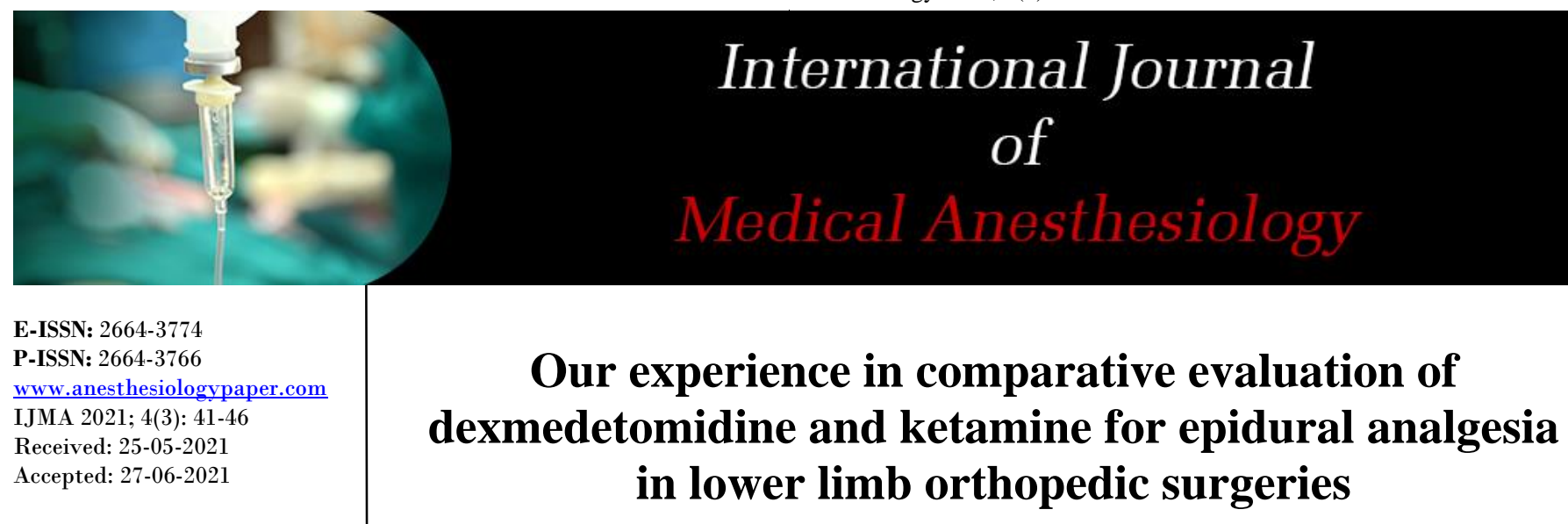

\section{Swathi P}

Senior Consultant Anaesthesiologist, Apple Hospital, Tanuku, Andhra Pradesh, India

Deepika E

Consultant Anaesthesiologist, Apple hospital, Tanuku, Andhra Pradesh, India

Sreerama Chandrudu A Consultant Anaesthesiologist, Apple hospital, Tanuku, Andhra Pradesh, India
Corresponding Author: Swathi P

Senior Consultant Anaesthesiologist, Apple Hospital, Tanuku, Andhra Pradesh, India

\section{Swathi P, Deepika E and Sreerama Chandrudu A}

\author{
DOI: https://doi.org/10.33545/26643766.2021.v4.i3a.280
}

\begin{abstract}
Introduction: The combined spinal epidural anesthesia (CSE) plays a unique role in management of perioperative analgesia. It is associated with early postoperative mobilization and rehabilitation with minimally associated pain and discomfort, which is the most desirable feature in modern surgery. We designed this study for comparative evaluation of dexmedetomidine and ketamine for epidural analgesia in lower limb orthopedic surgeries in patients operated under CSE.

Materials and Methods: The study included 50 patients divided into two groups ( 25 each). Group D: Patient received bolus dose of dexmedetomidine $1 \mu \mathrm{g} / \mathrm{kg}$ after spinal anesthesia followed by bupivacaine $0.125 \%$ with dexmedetomidine $1 \mu \mathrm{g} / \mathrm{ml}$ epidurally using $240 \mathrm{ml}$ elastomeric pump at $5 \mathrm{ml} /$ hr till 48 hours. Group K: Patient received bolus dose of ketamine $0.5 \mathrm{mg} / \mathrm{kg}$ after spinal anesthesia followed bupivacaine $0.125 \%$ with ketamine $0.5 \mathrm{mg} / \mathrm{ml}$ epidurally using elastomeric pump at $5 \mathrm{ml} / \mathrm{hr}$ till 48 hours.

Results: There was no significant difference between the two groups $(p>0.05)$ in terms of highest sensory level achieved and time for complete motor block. The patients in both the groups were hemodynamicaly stable throughout the study. The duration of analgesia was longer with dexmedetomidine however, none of the patients in both the groups required rescue analgesic. The time to regression of sensory level to L5 in Group D was found to be $594 \pm 63.04$ minutes as compared to $362.4 \pm 45.76$ minutes in Group K $(p<.001)$ while, the time to regression of motor block to Bromage scale 1 in Group D was $488.4 \pm 42.88$ minutes whereas, in Group K it was $303.6 \pm 36.04$ minutes $(p<0.001)$. The incidence of sedation, nausea, vomiting and other side effects were comparable.

Conclusion: We conclude that continuous epidural infusion with dexmedetomidine and ketamine effectively reduced the postoperative rescue analgesics requirements and provided good patient satisfaction in patients undergoing lower limb orthopedic surgery.
\end{abstract}

Keywords: dexmedtomedine, ketamine, epidural

\section{Introduction}

The International Association for the Study of Pain (IA SP) has rightly defined pain as "an unpleasant sensory and emotional experience associated with actual or potential tissue damage, or described in terms of such damage." The orthopaedic surgeries are characterised by early intense postoperative pain and thus, are associated with high demand for analgesics including opioids. The modern orthopaedics is about early postoperative mobilisation and rehabilitation along with minimal pain and discomfort for better results ${ }^{[1]}$. The use of neuraxial blocks for orthopaedic lower limb surgeries has increased rapidly due to increasing demand for postoperative pain relief and also to decrease the requirement of intravenous analgesics. Among various techniques available for neuraxial blockage, combined spinal epidural (CSE) anaesthesia has unique advantage of flexibility of continuous epidural block which help to titrate desired sensory level, vary the block intensity, control duration of anaesthesia and deliver postoperative analgesia ${ }^{[2]}$. The use of epidural analgesia for the management of post-operative pain has evolved as critical component of multimodal approach to achieve the goal of adequate analgesia with improved outcome. In addition to improved pain control, epidural analgesia can improve patient outcome by attenuating detrimental perioperative physiology ${ }^{[3,4]}$. One of the major advantages of epidural analgesia is that it facilitates early postoperative mobilisation and rehabilitation with minimally associated pain and discomfort. This will help patient in terms of shorter hospital stay and decreased expenditure. 
This is highly important in terms of physical, psychological and economic factors [5, 6]. Many a times for achieving desired peri-operative anesthetic effect, invariably large volumes of local anesthetics are used, thereby increasing the possibilities of local anesthetic toxicity and deleterious haemodynamic consequences. In neuraxial anaesthesia, the use of local anaesthetic agents can be minimize by adding adjuvants having ideal qualities like sedation, stable haemodynamic and capability to provide smooth and longer intraoperative and post-operative analgesia [7]. Various adjuvants such as opioids, adrenaline, sodium bicarbonate, $\alpha-2$ adrenoreceptor agonists and GABA receptor agonists are used to improve and prolong analgesia ${ }^{[8]}$. Dexmedetomidine is a new addition to the class of $\alpha 2$ adrenergic agonists which has got numerous beneficial effects when used as adjuvant through epidural route. It acts on both pre and post synaptic sympathetic nerve terminal and central nervous system thereby decreasing the sympathetic outflow and norepinephrine release. Thus, it has sedative, anxiolytic, analgesic, sympatholytic and stable hemodynamic effects. Dexmedetomidine does cause a controlled hypotension and bradycardia but main feature of this drug is the lack of opioids related side effects like pruritus, nausea, vomiting and respiratory depression ${ }^{[5,9]}$. The NMDA receptors are ligand gated ion channels in dorsal horn of spinal cord, which gets activated on tissue injury and thus cause central sensitisation and severe pain. NMDA receptor antagonists inhibit hyperalgesia caused by inflammation, tissue and nerve injury. Ketamine is an $\mathrm{N}$ methyl-D-aspartate (NMDA) receptor antagonist whose popularity in controlling pain is on the rise. Ketamine as an adjuvant non-competitively antagonise them, hence potentiating the effect of local analgesics ${ }^{[3]}$. The purpose of this study was to compare the efficacy of these two adjuvant drugs along with bupivacaine for epidural analgesia in lower limb orthopedic surgeries under combined spinal epidural anesthesia.

\section{Materials and Methodology \\ Aim}

To compare the effects of dexmedetomidine and ketamine as an adjuvant to bupivacaine for continuous epidural infusion in lower limb orthopaedic surgeries, regarding block characteristics, haemodynamic changes, sedation, post-operative analgesia and side effects if any.

\section{Materials and Methods}

Study was carried out at Apple hospital, Tanuku. Written informed consent from patients was taken. 50 patients of either sex, age group (18-65 years), ASA grade I and grade II scheduled for lower limb surgery under combined spinal plus epidural anaesthesia were included in this study and divided into two groups of 25 each. The duration of study was of 18 months starting form March 2018 to September 2020. Patients refusing to participate, patients with any contraindication to spinal anaesthesia or allergic to local anaesthetic and study drugs, uncontrolled hypertension and bradycardia, patients with known neurologic and psychiatric illness were excluded from the study.

Thorough preanaesthetic evaluation was carried out on the previous day of surgery and patients were explained regarding the procedure and assessment of pain using the Visual Analogue Scale [VAS 0-10]. Basic laboratory investigations like complete haemogram, blood sugar, renal function test, urine routine/micro etc. were reviewed. ECG and chest radiogram reviewed in all the patients. All patients were kept nil by mouth minimum for six hours prior to anaesthesia. On arrival in the recovery room, preoperative baseline pulse rate, blood pressure and VAS score were recorded. Intravenous line was secured and preloading was done with an infusion of Ringer's lactate $10 \mathrm{ml} / \mathrm{kg}$. Premedication was given by intramuscular route half an hour before surgery as inj. Glycopyrrolate $0.004 \mathrm{mg} / \mathrm{kg}$ and inj. Midazolam $0.04 \mathrm{mg} / \mathrm{kg}$. All patients received combined spinal epidural anaesthesia. The Tuohy $18 \mathrm{G}$ Epidural needle was inserted under all aseptic and antiseptic precautions in the sitting position in L2-L3 intervertebral space. Epidural space was located and confirmed by loss of resistance technique. Epidural catheter was inserted and secured at 5 $\mathrm{cm}$ into epidural space. Spinal anaesthesia was given at L3L4 intervertebral space with $25 \mathrm{G}$ spinal needle using 3-4ml, $0.5 \%$ Bupivacaine heavy. Patient was positioned in supine position and epidural bolus dose was given as per the group allotted. The patients were divided into either of the two groups:

1. Group D: Patient received bolus dose of dexmedetomidine $1 \mu \mathrm{g} / \mathrm{kg}$ diluted into $5 \mathrm{ml} \mathrm{NS}$ epidurally and after 1 hour they received bupivacaine $0.125 \%$ with dexmedetomidine $1 \mu \mathrm{g} / \mathrm{ml}$ epidurally using $240 \mathrm{ml}$ elastomeric pump at $5 \mathrm{ml} / \mathrm{hr}$ till 48 hours.

2. Group K: Patient received bolus dose of ketamine 0.5 $\mathrm{mg} / \mathrm{kg}$ diluted into $5 \mathrm{ml} \mathrm{NS}$ epidurally and after 1 hour they received bupivacaine $0.125 \%$ with ketamine $0.5 \mathrm{mg} / \mathrm{ml}$ epidurally using $240 \mathrm{ml}$ elastomeric pump at $5 \mathrm{ml} / \mathrm{hr}$ till 48 hours.

The highest level of sensory block achieved and time to complete motor block were recorded. The haemodynamics, sedation (Ramsay Sedation score 1-6) and pain scores (VAS 0 -10) were monitored at 0 minutes, $1 / 2$ an hour and then, hourly intraoperatively followed by every 2 hours till 12 hours and finally at 24 hours and 48 hours postoperatively. Time to sensory regression at L5 (pin prick method) and motor regression to Bromage scale 1 was recorded. Patients were to receive Inj. Bupivacaine $(0.125 \%) 1.5 \mathrm{ml} /$ segment as rescue analgesic, when pain persisted with $\mathrm{VAS} \geq 4$. The duration of surgery, total amount of rescue analgesic required and adverse effect, if any was noted. The epidural cathetar was removed after 48 hours postoperatively. Hypotension was defined as systolic blood pressure (SBP) $20 \%$ of baseline blood pressure and was corrected with fluids or injection mephentermine. Bradycardia was defined as heart rate $[\mathrm{HR}]<60 \mathrm{pulse} / \mathrm{min}$ was treated with injection atropine $0.3-0.6 \mathrm{mg}$ intravenously. 


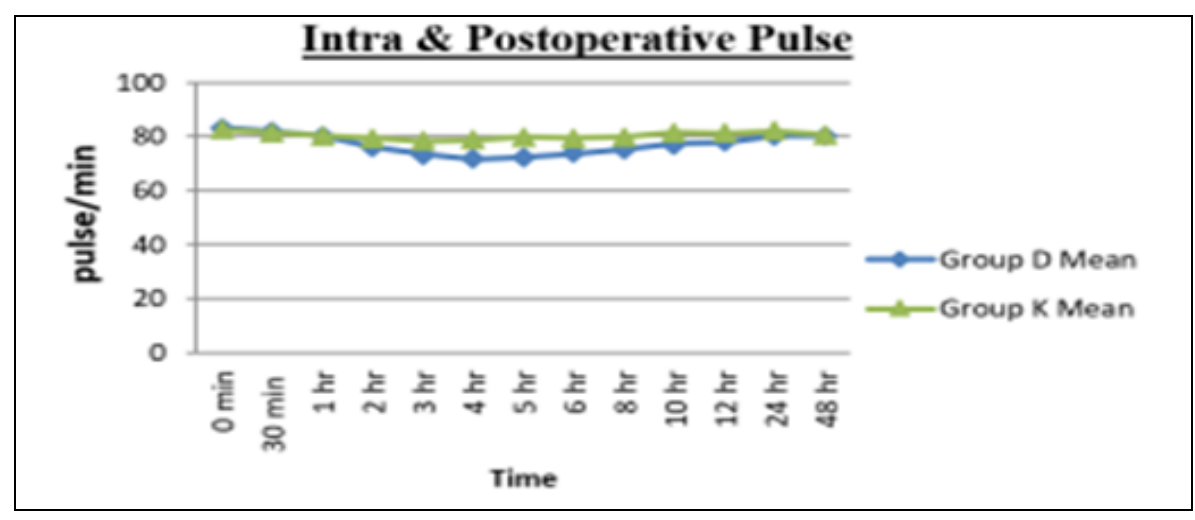

Fig 1: Mean pulse rate intra and postoperatively

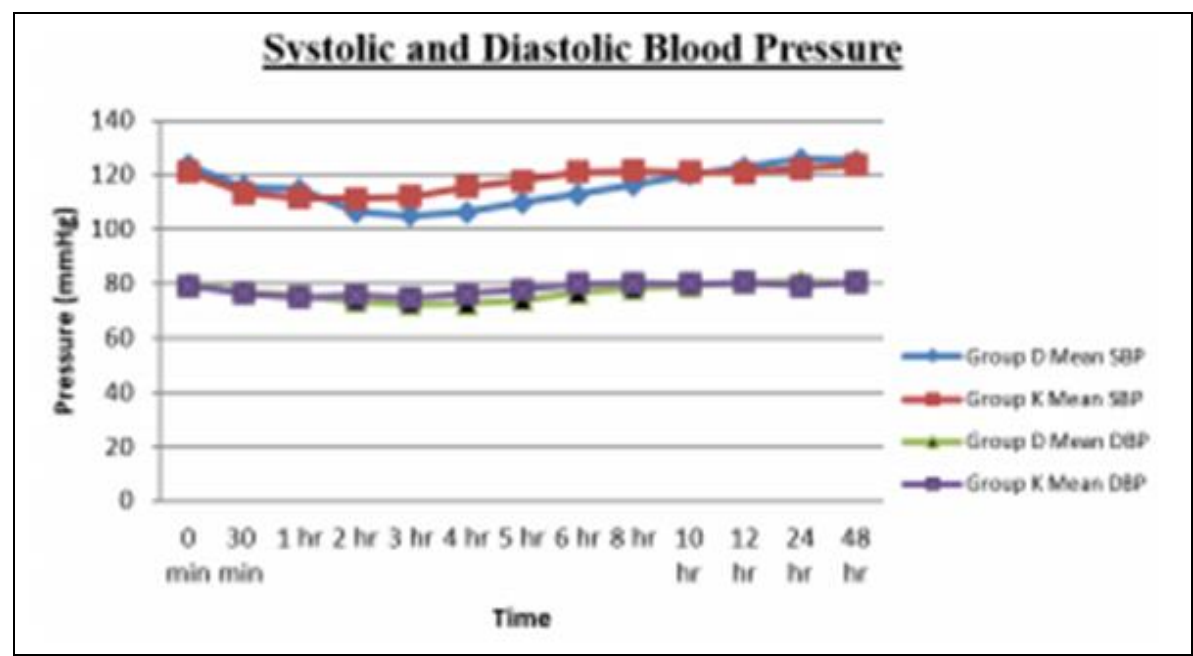

Fig 2: Mean systolic blood pressure (SBP) and diastolic blood pressure (DBP) intra and postoperatively

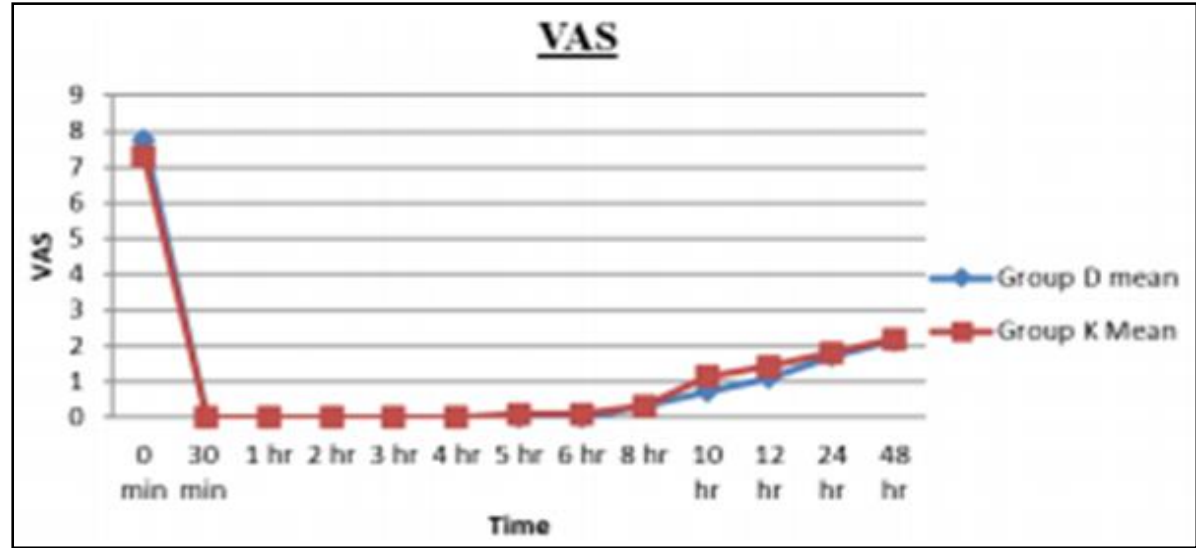

Fig 3: Mean visual analogue scale (VAS) intra and postoperatively

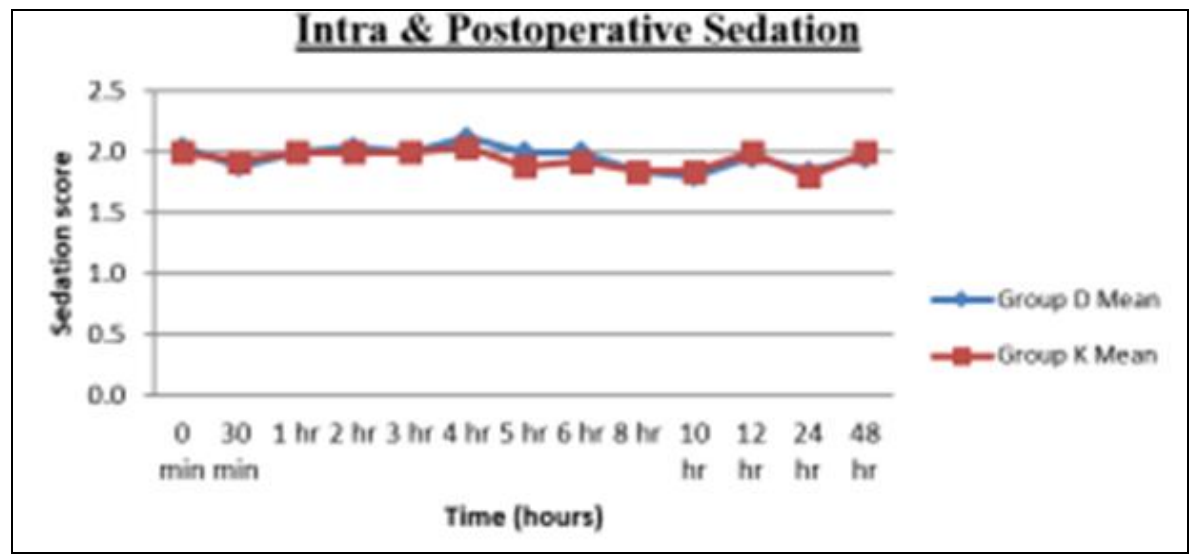

Fig 4: Mean intra and postoperative sedation score 


\section{Statistical Analysis}

Data was analyzed using OpenEpi software. The statistical analysis was done by unpaired t-test for quantitative data and chi-square test for qualitative data.

\section{Observation, Results \& Discussion}

One of the main concerns of anaesthesiologist is control of pain perioperatively as inadequate pain relief has adverse effects on patient's physiological, psychological and metabolic status. The combined spinal epidural anesthesia (CSE) plays a unique role in management of perioperative analgesia. Epidural in most commonly used mode on postoperative analgesia for lower limb surgeries including fixations and joint replacements. Dexmedetomidine is highly selective $\alpha 2$-adrenergic receptor agonist with sedative, anxiolytic, analgesic, antihypertensive and sympatholytic properties. Ketamine is a NMDA receptor antagonist which inhibits central sensitisation due to peripheral nociception. Epidural infusion of dexmedetomidine was used at a dose of $1 \mu \mathrm{g} / \mathrm{ml}$ as studied by Nilesh Balu Sonawane et al (2016), ${ }^{[10]}$ SJ Bajwa et al $2011^{[5]}$ and Nirmeen Fatima et al ${ }^{[8]}$ without any significant haemodynamic side effects, while, we used preservative free ketamine in concentration of $0.5 \mathrm{mg} / \mathrm{ml}$ for epidural infusion as studied by Nilesh Balu Sonawane et al (2016) ${ }^{[10]}$ without any neurotoxic adverse effects. The demographic profile of patients in both the groups was comparable in terms of weight and sex. In our study we included patients in age group of $18-65$ years. Mean age in Group D was $46.3 \pm$ 15.7 years while in Group $\mathrm{K}$ was $37.0 \pm 12.3$ years. This difference was statistically significant $(p>0.05)$ but as patients were not in extremes of age, it should not affect the outcome of study. Also patients were selected randomly so difference is acceptable. The mean duration of surgery in Group D was $152.4 \pm 51.2 \mathrm{~min}$ and in Group K was $139 \pm$ $33.3 \mathrm{~min}$. The maximum duration of surgery was $285 \mathrm{~min}$ in one patient of Group D, but patient didn't require any intraoperative top-up dose. There was statistically significant difference in mean duration of surgeries between the two groups ( $p$ 0.05). The mean time to achieve complete motor block in Group D was $11.36 \pm 0.99$ minutes as compared to $12.10 \pm 1.04$ minutes in Group K. This difference was statistically insignificant $(p>0.05)$. The findings of the present study were consistent with the

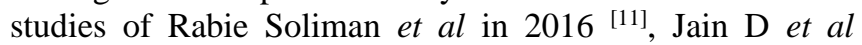
(2011) ${ }^{[3]}$, Nirmeen Fatima and colleagues (2018) ${ }^{[8]}$. However, in study conducted by SJ Bajwa et al in 2011 [5] comparing dexmedetomidine with fentanyl for epidural analgesia in lower limb orthopaedic surgeries, the time to reach complete motor block was shorter in patients receiving Dexmedetomidine (18.16 \pm 4.52 minutes) as compared to Fentanyl group (22.98 \pm 4.78 minutes) $(p<0.05)$. Therefore, difference in time to achieve complete motor between two groups is significant. Thus, findings of this study are inconsistent with our study. In present study, baseline $(0 \mathrm{~min})$ pulse rate, systolic blood pressure (SBP) and diastolic blood pressure (DBP) was comparable between both the groups $(P>0.05)$. Thereafter during observation period, reduction in pulse rate and blood pressure was observed in both the groups. However, reduction in pulse rate was more in Group D as compared to Group $\mathrm{K}$ from 4th -8 th hour of study period and difference was statistically significant (p 20\% of baseline value) which required treatment. Rabie Soliman et al in $2016^{[11]}$ studied dexmedetomidine and fentanyl as an epidural adjuvant for postoperative pain relief in patients undergoing total knee replacement. They found that the decrease in heart rate and mean blood pressure was significantly higher in dexmedetomidine group as compared to fentanyl group ( $\mathrm{p}$ 0.05 ). None of the patients had VAS score $\geq 4$ throughout the observation period; hence no rescue analgesic was required in any patient of the two groups. In 2013 Saravana Babu et al compared epidural dexmedetomidine with clonidine and found that VAS score were higher in clonidine group however, the difference was statistically insignificant $(p>0.05) .{ }^{[13]}$ Sarabjit kaur et al compared the effects of ropivacaine vs dexmedetomidine plus Ropivacaine. They observed that VAS score and requirement of rescue analgesia in patients receiving dexmedetomidine was significantly less than control group. In 2011 Mamta Sethi et al studied the role of epidural ketamine and reported that the VAS scores during rest and movement were constantly lower in ketamine group as compared to control group $(p<0.05) .{ }^{[3]}$ The analgesic effect of bupivacaine was potentiated by both, Dexmedetomidine and ketamine. The local anesthetics act by blocking sodium channels, while $\alpha 2$ - agonists bind to presynaptic C- fibres and postsynaptic dorsal horn, blocking the neurotransmission from Cfibres and by hyperpolarising the po stsynaptic neurons. 14 Activation of NMDA receptors due to inflammation, nerve and tissue injury leads to hyperexcitability of dorsal horn neurons causing central sensitisation and severe pain. Ketamine is a NMDA receptor antagonist, thus, it blocks the ascending transmission of nociceptive stimuli from dorsal horn neurons and provides satisfactory analgesia. ${ }^{[3]}$ Dexmedetomidine and ketamine are known to cause sedation. We assessed perioperative sedation using Ramsay sedation score and observed that difference in average sedation scores of the two groups were statistically insignificant throughout the observation period. None of the patient suffered from profound sedation requiring discontinuation of epidural infusion. The findings of our study were consistent with the studies conducted by MS Sarvana Babu et al and Hanan Shobary et al. However, in studies of Nirmeen Fatima et al with dexmedetomidine and Xing Xue et al with ketamine, patients experienced deep sedation at grade 4 or 5 . The sedative effect of Dexmedetomidine is due to hyperpolarisation of noradrenergic neurons in locus ceruleus of brain stem leading to inhibition of noradrenaline re lease which has hypnotic effect while, ketamine acts by antagonising the NMDA receptors present in locus ceruleus which modulates the noradrenergic and serotonergic neurons consequently causing sedation. ${ }^{[15]}$ The sedation caused by both the drugs is profound when given through intravenous route, but in our study we administered it epidurally which leads to decrease bioavailability and hence, mild sedation only. ${ }^{[14]}$ The mean time to regression of sensory level to L5 in Group $\mathrm{D}$ was found to be $594 \pm 63.04$ minutes as compared to $362.4 \pm 45.76$ minutes in Group $\mathrm{K}(p<0.001)$. The time to regression of motor block to Bromage scale 1 in Group D was $488.4 \pm 42.88$ minutes whereas, in Group $\mathrm{K}$ it was $303.6 \pm 36.04$ minutes $(p<0.001)$. This suggests that sensory and motor block in patients receiving dexmedetomidine was markedly prolonged. In study conducted by Nilesh Sonawane et al in 2016 receding time of sensory block in dexmedetomidine group was $9.33 \pm 4.34$ hours as compared to $7.03 \pm 3.79$ hours in ketamine group $(p<0.05)$. The 
receding time of motor blockade in dexmedetomidine group was $7.10 \pm 3.53$ hours while in ketamine group it was $3.80 \pm$ 1.49 hours $(p<0.001)$. Similar findings were observed by Sarabjit Kaur et al, Safiya Shaikh et al and SJ Bajwa et al. The major site of action for $\alpha 2$-agonist is dorsal horn neurons of spinal cord where it caused inhibition of neurotransmission through $\mathrm{C}$ and $\mathrm{A} \delta$ fibres and hence the effect. ${ }^{[14]}$ Ketamine inhibits the NMDA receptors in the spinal cord which facilitates the transmission of nociceptive stimuli and thus, affects central sensitisation. The side effects profile of the two study drugs was quite comparable. Nausea occurred in $3(12 \%)$ patients in Group D and in 1 (4\%) patient of Group K, which is statistically insignificant. There was no recorded case of vomiting. Our finding were similar to that of other studies. 5,10,12 We observed that 1 (4\%) patient in dexmedetomidine group suffered from significantly profound motor block of 14 hours which required discontinuation of infusion pump.

The patient recovered well without any neurological deficit. Nilesh Sonawane et al ${ }^{[10]}$ reported similar side effect with Dexmedetomidine. None of the patient suffered from profound hypotension and bradycardia, which are known side effects of dexmedetomidine. The use of ketamine is associated with neurological effects like hallucination and delirium. However we didn't report a single case of such side effects. It may be due to use of lower dose and the epidural route of administration. These findings were in agreement with previous observations made by Hanan Shobary et al, Alireza Kamali et al ${ }^{[6]}$ and Mamta Sethi et al. ${ }^{[3]}$ Limitations of our study are relatively small number of patients studied and availability of only few studies carried out with ketamine for CSE with very few parameters observed, which makes it difficult to compare and evaluate our findings.

Table 1: Demographic data

\begin{tabular}{|llll|}
\hline Particulars & Group D & Group K & P value \\
& Mean \pm SD & Mean \pm SD & \\
Age(years) & $46.3 \pm 15.7$ & $37.0 \pm 12.3$ & $\mathrm{P}<0.05$ \\
Weight(kg) & $60.6 \pm 8.33$ & $60.5 \pm 8.24$ & $\mathrm{P}>0.05$ \\
Gender(M/F) & $17 / 8$ & $19 / 6$ & $\mathrm{P}>0.05$ \\
Duration of Surgery(min) & $152.4 \pm 51.2$ & $139 \pm 33.3$ & $\mathrm{P}<0.05$ \\
\hline
\end{tabular}

Table 2: Block characteristics

\begin{tabular}{|c|c|c|c|}
\hline Particulars & Group D & Group K & P Value \\
\hline $\begin{array}{l}\text { Highest sensory block level achievedNo. } \\
\text { of patients (\%)T8 T10 }\end{array}$ & $17(68) 8(32)$ & $16(64) 9(36)$ & $>0.05>0.05$ \\
\hline Time for complete Motor Block (min) & $11.36 \pm 0.99$ & $12.10 \pm 1.04$ & $>0.05$ \\
\hline $\begin{array}{l}\text { Time to regression of Sensory level to L5 } \\
(\min )\end{array}$ & $594 \pm 63.04$ & $362.4 \pm 45.76$ & $<0.001$ \\
\hline $\begin{array}{l}\text { Time to regression of motor block to } \\
\text { Bromage scale } 1 \text { (min) }\end{array}$ & $488.4 \pm 42.88$ & $303.6 \pm 36.04$ & $<0.001$ \\
\hline
\end{tabular}

Table 3: Comparison of side-effects

\begin{tabular}{|lllll|}
\hline Side Effects & Group D (n=25) & \multicolumn{3}{c|}{ Group K (n=25) } \\
Nausea & No. & $\%$ & No. & $\%$ \\
Vomiting & 3 & $12 \%$ & 1 & $4 \%$ \\
Hypotension & & & & \\
Bradycardia & & & \\
Dry Mouth & & & \\
Significantly profound Motor Block & 1 & $4 \%$ & \\
Hallucination & & & \\
Delirium & & & & \\
\hline
\end{tabular}

\section{Conclusion}

We concluded that ketamine administered epidurally, prolonged the duration of analgesia, less than the epidural dexmedetomidine. However, both the drugs effectively reduced the postoperative rescue analgesics requirements and provided greater patient satisfaction. So, continuous epidural infusion with dexmedetomidine and ketamine can be a promising technique for pain management in patients undergoing lower limb orthopedic surgery

\section{Conflict of Interest}

None declared 


\section{References}

1. Karhade SS, Acharya SA, Harnagale K. Comparative analysis of epidural bupivacaine versus bupivacaine with dexmedetomidine for vaginal hysterectomy. Anesth, Essays Res 2015;9(3):310-313.

2. Morgan, Ikhail's. Clinical anesthesiology. In: Clinical anesthesiology, fifth Edition (McGraw Hill Education), chapter 45. Spinal, Epidural \& Caudal Blocks 937$974 \mathrm{p}$.

3. Sethi M, Sethi N, Jain P, Sood J. Role of epidural ketamine for postoperative analgesia after upper abdominal surgery. Indian J Anesth 2011;55(2):141145.

4. Shaikh SI, Revur LR, Mallappa M. Comparison of Epidural Clonidine and Dexmedetomidine for Perioperative Analgesia in Combined Spinal Epidural Anesthesia with Intrathecal Levobupivacaine: A Randomized Controlled Double-blind Study. Anesth Essays Res 2017;11(2):503-507.

5. Bajwa SJS, Arora V, Kaur J, Singh A, Parmar SS. Comparative evaluation of dexmedetomidine and fentanyl for epidural analgesia in lower limb orthopedic surgeries. Saudi J Anesthesia 2011;5(4):365-370.

6. Kamali A, Zarei A, Moshiri E, Naziri M. Comparing the Effect of Adding Ketamine and Neostigmine to 0.25 $\%$-Bupivacaine for Epidural Analgesia in Patients Candidate for Elective Surgery of Femur Fracture. JSSU 2014;22(5):1464-1471.

7. Singh BS, Bajwa Sukhminder Kaur et al. Dexmedetomidine and clonidine in epidural anaesthesia: A comparative evaluation. Indian J Anaesth 2011;55(2):116-121.

8. Fatima N, Singh NR, Singh LPK, Doddaiah DB, Singh TH, Taloh Y. Comparative study of the effect of dexmedetomidine and butorphanol as epidural adjuvants in abdominal hysterectomy under intrathecal levobupivacaine anesthesia. J Med Soc 2016;30(3):166171. doi:10.4103/0972-4958.191183.

9. Paula, Saraiva PF. Synergistic effect between dexmedetomidine and $0.75 \%$ ropivacaine in epidural anesthesia. Rev Assoc Med Bras 2008;54:110-115.

10. Sonawane NB, Balavenkatasubramanian J, Gurumoorthi P, Jadhav PA. Quality of post-operative analgesia after epidural dexmedetomidine and ketamine: A comparative pilot study. Indian J Anesthesia 2016;60(10):766-768.

11. Soliman R, Eltaweel M. Comparative study of dexmedetomidine and fentanyl as an adjuvant to epidural bupivacaine for postoperative pain relief in adult patients undergoing total knee replacement: a randomized study. J Anesthesiol Clin Sci 2016;5:1-1.

12. Xue X, Lv Y, Zhao Y, Leng Y, Zhang Y. Efficacy of prophylactic epidural ketamine for reducing shivering in patients undergoing caesarean section with combined spinal-epidural anesthesia. Biomedical Reports 2018;8(5):485-490.

13. Babu S, Kumar VA. A comparative study in the postoperative spine surgeries: epidural ropivacaine with dexmedetomidine and ropivacaine with clonidine for post-operative analgesia. Indian $\mathbf{J}$ Anaesth 2013;57(4):371-376.

14. Stoelting K. Pharmacology and physiology in Anesthesia practice. Anesthesiology textbook 4th edition (Lippincott Williams \& Wilkins); Ch-15,
Antihypertensive drugs: 344-345, Ch-3, opioid agonists and antagonists.

15. Jain D, Khan RM, Kumar D. Perioperative effect of epidural dexmedetomidine with intrathecal bupivacaine on haemodynamic parameters and quality of analgesia. South Afr J Anaesth analg 2012;18(2):105-109. 